\title{
Molecular dynamics study of the effect of induced mutations on the protein structures associated with diseases of a radiobiological nature
}

\author{
Kholmirzo T. Kholmurodov ${ }^{1,2, *}$ \\ ${ }^{1}$ Laboratory of Radiation Biology, Joint Institute for Nuclear Research \\ ${ }^{2}$ Dubna International University, Dubna, Moscow Region, Russia
}

\section{Email address:}

mirzo@jinr.ru (K. T. Kholmurodov)

\section{To cite this article:}

Kholmirzo T. Kholmurodov. Molecular Dynamics Study of the Effect of Induced Mutations on the Protein Structures Associated with Diseases of A Radiobiological Nature. American Journal of Bioscience and Bioengineering. Vol. 1, No. 1, 2013, pp. 7-16. doi: 10.11648/j.bio.20130101.12

\begin{abstract}
The induced mutations in biological molecules, such as DNA and proteins, have quite a different nature (environmental factors, viruses, ionizing radiation, mutagenic chemicals, inherited genetic alterations, etc.). Induced mutations can destroy the existing chemical (hydrogen) bonds in the native molecular structures or, on the contrary, create new chemical (hydrogen) bonds that do not normally exist there. In protein structures, the cause of such changes might be the substitution of one or several specific amino acid residues (point mutations). At the atomic level, the replacement of one amino acid residue by another causes essential modifications of the molecular force fields of the environment, which can break important hydrogen bonds underlying the structural stability of biological molecules. In this work, based on molecular dynamics (MD) method, we demonstrate the effect of mutational structure changes on several biological protein models (the p53 oncoprotein, visual pigment rhodopsin, cyclin-dependent kinase, and recA protein). Molecular dynamics simulation is a powerful tool in investigating the structure properties of biological molecules on the atomic and molecular levels, and it has been widely used to study the structural conformational behavior of proteins. We also discuss the scenario of the mutation effects associated with different kinds of diseases that could develop and take place in physiological conditions.
\end{abstract}

Keywords: Molecular Dynamics, Structure Conformation, Proteins, Mutations, Diseases

\section{Introduction}

The objects of radiation genetics and radiobiology research include the mechanism of the induction of mutations of different nature by ionizing radiation. High-power beams of particle accelerators provide a wide basis for the investigation of the mutagenic effect of ionizing radiation. In some aspects, computer molecular simulation and analysis is an extremely efficient tool for supporting various biophysical and radiobiological experiments. Specially mentioned should be the modern molecular dynamics approach, which is widely applied in todays biophysical, radiobiological, and materials science research. Based on advanced molecular dynamics methods and visualization techniques, we simulate here the behavior of wild-type and mutated proteins in water and ionic solvents at physiological conditions. The obtained molecular dynamics (MD) simulation results are discussed in terms of their strong correlation with different diseases of a radiobiological nature [1-4].

Changes in the functions of proteins can originate from their improper conformational behavior and unfolding. The cause of such changes might be a substitution of one or several specific amino acid residues in the protein structure. At the atomic or molecular level, the replacement of one amino acid residue by another causes essential modifications of the molecular force fields of the environment and can break important hydrogen bonds underlying the structural stability of biological molecules. As a result, we get global structure changes in biomolecules, which make their functional behavior different than that of the native ones. In other words, a specific disease (say, cancer) can develop if a biomolecule (protein) becomes unable to perform its function.

Experimental studies of mutation transitions in protein structures with traditional X-ray or NMR techniques are an extremely difficult, time- and money-consuming task. Using proper computational methods (molecular dynamics 
(MD)) and efficiently implementing them on parallel/vector or special-purpose MD machines, it is possible to perform an adequate study. In the following sections, we demonstrate an efficient use of molecular simulation for a number of radiobiological objects (the p53 oncoprotein, visual pigment rhodopsin, cyclin-dependent kinases, and RecA proteins) [2-14].

\section{The P53 Oncoprotein: the Effect of the Arg273 $\rightarrow$ His Mutation on the DNA Binding Domain}

The p53 protein (the $53(\mathrm{kDa})$ kilodalton protein) is activated either to induce a cell cycle arrest allowing the repair and survival of the cell, or apoptosis to discard the damaged cell (the structure of the mouse protein p53 is shown in Figure 1).

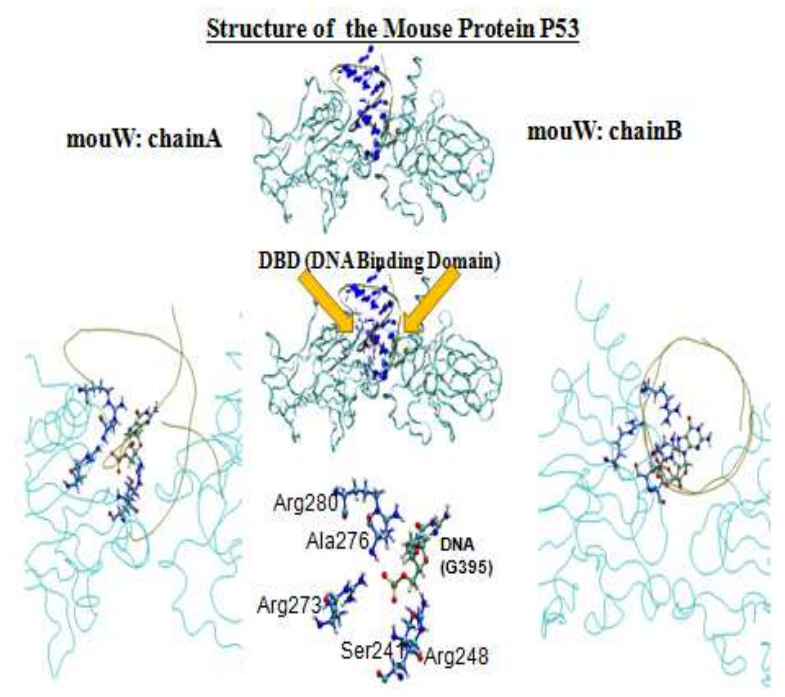

Figure 1. The side and top views of the 553 mouse protein and $D B D$ (DNA-binding domain). For the p53 protein structure, two chains (A and B) are shown.

The p53 tumor suppressor protein is involved in preventing cancer and plays a central role in conserving genomic stability by preventing a genome mutation. Single amino acid substitutions (mutations) in the p53 structure deactivate the p53 protein, which results in cancer. Most of the mutations (95\% of all known tumor mutations) usually occur in so-called DBD (the DNA-binding domain) of the p53 protein. Thus, an oncogenic form of p53 is predominantly a full-length $\mathrm{p} 53$ protein with a single amino acid substitution in the DBD. Most of these mutations destroy the ability of the protein to bind to its target DNA sequences, and thus prevent the transcriptional activation of these genes. It is worth noting that tumors with inactive p53 mutants are aggressive and often resistant to ionizing radiation and chemotherapy.

Separately, in Figure 2, we display for the mouse p53 protein a picture of the mutational amino acid exchange Arg273 $\rightarrow$ His in the DBD contact region. In Figure 2 the positions of two closest to the mutation point of DNA phosphorus atoms, P(DG395) and P(DT394), are shown. It is worth noting that arginine (Arg) is an alpha-amino acid with $\mathrm{pKa}=12.48$; its side chain consists of a 3-carbon aliphatic straight chain and is positively charged in neutral, acidic, and even most basic environments. Because of conjugation between the double bond and the nitrogen lone pairs, the positive charge is delocalized, enabling the formation of multiple H-bonds. Histidine (His) is an aromatic amino acid with $\mathrm{pKa}=6.5$; its side chain consists of a positively charged imidazole ring which is aromatic at all $\mathrm{pH}$ values. This means that at physiologically relevant $\mathrm{pH}$ values, relatively small shifts in $\mathrm{pH}$ will change its average charge. Below a $\mathrm{pH}$ of 6 , the imidazole ring is mostly protonated. The differences in the chemical structure and properties between arginine and histidine during nanosecond dynamical changes could influence the final (relaxed) states of all amino acids.

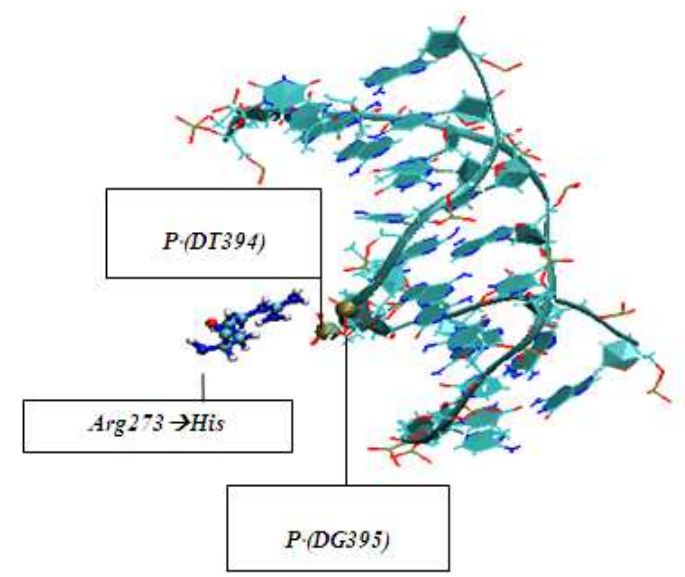

Figure 2. A snapshot of mutational amino acid exchange Arg273 $\rightarrow$ His for the DNA contact region is represented. The positions of two closest to Arg273 $\rightarrow$ His phosphorus atoms of DNA, P(DG395) and P(DT394), are also shown.

We have performed a comparative molecular dynamics (MD) simulation analysis between the wild-type and mutant versions of the mouse p53 proteins and have examined the effect of the Arg273 $\rightarrow$ His mutation on the p53-DNA binding domain. (The MD simulation details have been described below in the Appendix). In Figure $3(\mathrm{a}, \mathrm{b})$ the results of the MD distance calculations on $\mathrm{p} 53$ dynamical changes in the region of the $\operatorname{Arg} 273 \rightarrow$ His and DNA interaction are presented. Figure $3 \mathrm{a}$ shows the distance d[Arg273-P(DG395)] distribution for the native protein; Figure $3 \mathrm{~b}$ shows the same distance distribution $\mathrm{d}[$ His273-P(DG395)] but for the mutant p53 protein.

Thus, our MD simulation results on the structure of the p53 oncoprotein with Arg273 $\rightarrow$ His mutation suggest that the induced mutation essentially disturbs the stability of the p53 core domain. Comparing the relaxed configurations and distance distribution diagrams between the native (Figure 3(a)) and mutant (Figure 3 (b)) p53 proteins, we clearly see that the $\operatorname{Arg} 273 \rightarrow$ His mutation has a considerable effect on the p53 - DNA contact region. The 
induced $\operatorname{Arg} 273 \rightarrow$ His mutation in the p53 protein significantly changes the picture of the hydrogen bond formation: apparently, it can destroy the existing native hydrogen bond. This may inambiguously imply consiquent changes in the functioning of the protiein, since a correlation beween the protein function and structures is obvious. It is well known that concerning the physiological aspect, a protein molecule can perform an unique function depending on its structure specifics.

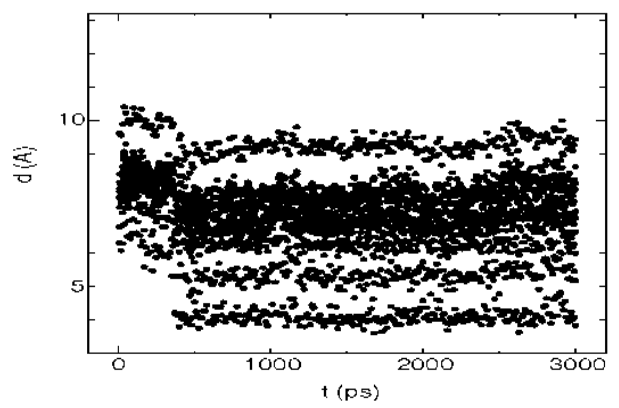

(a)

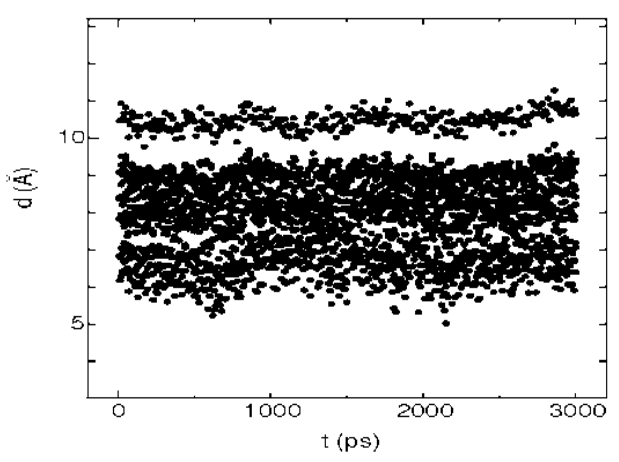

(b)

Figure 3(a,b). The distance distribution diagrams of the 3-ns dynamical changes between the wild-type (Arg273) and mutant (Arg273 $\rightarrow$ His) p53 mouse proteins for the p53-DNA contact region: (a) Arg273-P(DG395); (b) His273-P(DG395).

With regard to the p53 oncoprotein, some reported observations on the effect of the Gly245 $\rightarrow$ Ser, $\operatorname{Arg} 248 \rightarrow$ Gln, Arg249 $\rightarrow$ Ser, and Arg273 $\rightarrow$ His mutations suggest that they disturb essentially the stability of the p53 core domain. A detailed analysis of the p53 mutations shows that the vast majority of the mutations in $\mathrm{p} 53$ cluster in the conserved regions of the DNA-binding core domain (residues 96-292). Around $20 \%$ of all mutations are concentrated in five "hotspot" codons in the core domain: 175, 245, 248, 249, and 273. Experimental ob servations indicate that the point mutations in p53 occur at a rate of approximately $70 \%$ in hormone-refractory prostate cancer and that $\operatorname{Arg} 273 \rightarrow$ His mutation of p53 protein (p53Arg273His) facilitates androgen-independent growth in castrated nude mice, etc. In this aspect, a good correlation between our MD simulation results and experimental observations has clearly seen. Moreover, our MD simulation results illustrate a detailed molecular mechanism of the conformational changes of key amino acid residues in the p53-DNA binding domain, which might be important for the understanding of the physiological functioning of the $\mathrm{p} 53$ protein and the origin of cancer $[1-2,10]$.

\section{The Visual Pigment Rhodopsin with 11-cis Retinal: Rhodopsin's Glu181 $\rightarrow$ Lys Mutation and Retinitis Pigmentosa}

Rhodopsin is a typical member of the G-protein-coupled receptor family. This visual pigment is the only lightsensitive protein in the rod's outer segment photoreceptor membrane. It consists of a chromophore, 11-cis-retinal, and opsin - a seven-helical membrane protein (see Figure 4).

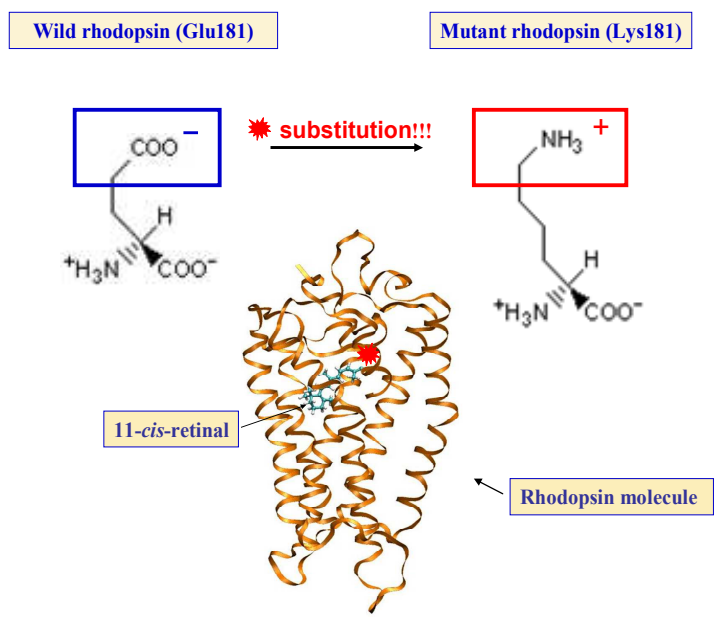

Figure 4. Wild-type and mutant (Glu181 $\rightarrow$ Lys) forms of the rhodopsin protein.

Retinitis pigmentosa (RP) is an inherited degenerative disease of the retina characterized by progressive photoreceptor degeneration, night blindness, visual field constriction, and electroretinographic abnormalities, with rods affected earlier than cones. This pathology can be very strong. The autosomal dominant form of retinitis pigmentosa (ADPR) leads to photoreceptor cell death and retina degeneration. Approximately $25 \%$ of this pathology is associated with the RP4(RHO)/Rhodopsin(3q) mutation of the rhodopsin gene. More than 100 mutations of this visual pigment are known. These mutations are found in all three rhodopsin domains (intradiscal, transmembrane, and cytoplasmic). 11-cis-retinal covalently binds to the protein through a protonated Schiff base (PSB) linkage to the $\varepsilon$ amino group of Lys296. In the dark-adapted state of rhodopsin, this PSB linkage is sufficiently stable. The amino acid substitution in the chromophore center during rhodopsin biosynthesis leads to the most distinctive clinical pathology of the ADPR. The consequence of mutations like these is protein misfolding. As a result, formation of stable PSB linkage between 11-cis-retinal chromophore and amino acid residue Lys296 is impossible.

We have analyzed the molecular dynamics (MD) of 
rhodopsin with the Glu181 $\rightarrow$ Lys point mutation to understand the molecular mechanisms of the retinal degeneration and retinitis pigmentosa origin. The results of our MD calculations for the rhodopsin protein with the mutation Glu181 $\rightarrow$ Lys are demonstrated in Figures 5. The MD simulation results indicate that the absence of the Glu amino acid in position 181 leads to the Ser186 replacement (compare Figures 5, A and B). This one moves towards Glu113 as in the case of free opsin (that is, rhodopsin without 11-cis-retinal). We could suggest that a weak hydrogen bond between the N-th atom of PSB and Glu113 is generated via Ser186. So, in the mutant Glu181 $\rightarrow$ Lys rhodopsin version, a situation occurs where a proton from PSB can easily break away, promoting Schiff base hydrolysis. In other words, the protonated Schiff base linkage becomes unstable. It leads to the impairment of the native visual pigment formation and, as a result, to the initiation of a photoreceptor cell pathology. Currently, there is no therapy that would stop RP development or restore vision; so, the vision prognosis is poor. Thus, understanding the molecular mechanisms of retinal degeneration caused by the above minimal structural changes in the photoreceptor has become an aim of modern research.

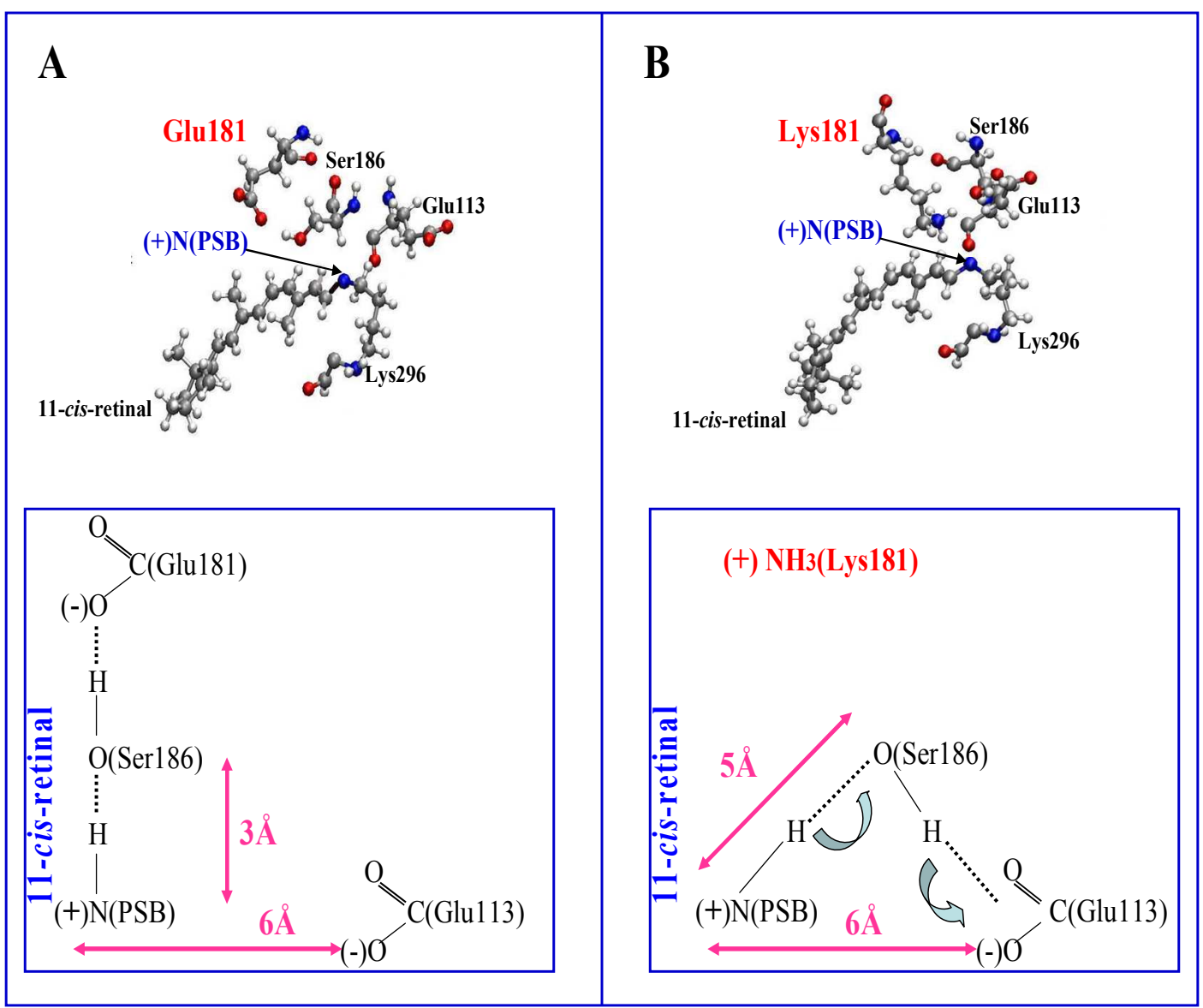

Figure 5. (Top) A snapshot of the molecular dynamics of amino acid residues Glu113, Glu181, and Ser186 in the region of protonated Schiff base linkage $((+) N P S B)$ for the wild-type rhodopsin $(A)$ and Glu181 $\rightarrow$ Lys mutant forms $(B)$. (Bottom) Diagrams of protonated Schiff base linkage stabilization in the wild (A) and mutant forms of rhodopsin (B).

Our MD simulation results illustrate in detail that in the case of the Glu181 $\rightarrow$ Lys point mutation, there is an electrostatic interaction disturbance between the 11-cis-retinal chromophore and surrounding amino acid residues within the chromophore centre of the rhodopsin molecule. As a result, the stable Schiff base linkage cannot be generated, and a rhodopsin molecule cannot be formed. A disturbance of electrostatic interactions of the 11-cis-retinal chromophore with surrounding amino acid residues leads to disorder in cytoplasmic loop packing. Consequently, mutant rhodopsin can be permanently activated, which leads to cell death [2-3,15-19].

\section{The Cyclin-Dependent Proteinkinases CDK2: Analysis of Kinase - Cyclin A Interactions in The T-Loop Vicinity For The Gly16 $\rightarrow$ Ser and Arg274 $\rightarrow$ GIn Mutant Complexes}

The eukaryotic cycle is coordinated by several related Ser/Thr protein kinases consisting of a catalytic cyclindependent kinase (CDK) and regulatory cyclin subunits. The transient appearance of these CDK-cyclin complexes 
drives the cell cycle events. The CDK subunit is inactive as a protein kinase without the cyclin subunit. The CDKcyclin binding provides the kinase activity of the complex, followed by phosphorylation of residue Thr 160 in the Tloop and dephosphorylation of Thr14 in the G-rich loop, resulting in full CDK activity. The kinases catalyze the transfer of the $\gamma$-phosphate in the adenosine triphosphate (ATP) molecule to a protein substrate. The CDK2 (33 kDa) has a typical bilobal kinase fold with a N-terminus (residues 1-85), mainly consisting of a $\beta$-sheet and a $\mathrm{C}$ terminus (residues 86-208), which is composed mostly of $\alpha$-helices (Figure 6).

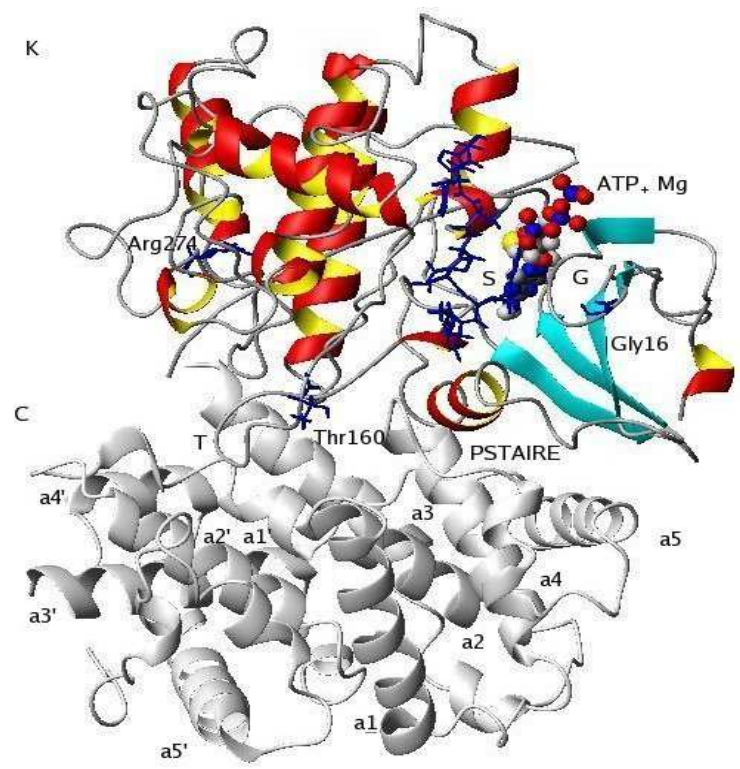

Figure 6. A ribbon structure of a protein kinase of the human CDK2. The protein complex consists of the kinase $(K)$ and cyclin $A(C)$ parts. The drawn bonds also show the amino acidic residues $T 160$ - a site of phosphorylation in a T-loop, Gly 16 - a site of the mutation substitution of glycine by serine; and Arg 274 - a site of the mutation substitution of arginine by glutamine.

We have simulated the conformational behavior of the CDK2 - cyclin A interface for the wild-type and two mutant forms of CDK2. The first mutation form of the CDK2 protein was a substitution Gly16 $\rightarrow$ Ser. This substitution is positioned on the third glycine of the conserved sequence GxGxxG in the G-rich loop. The G- and T-loops and PSTAIRE helix form a cleft where the ATP molecule is placed. The second mutation Arg274 $\rightarrow$ Gln is located in the C-terminus of a large kinase lobe which is far from the activating loops or CDK2 - cyclin A interface. Based on the simulation data for the equilibrated structures, we have performed a comparative analysis of the kinase structural changes in its active site. The behavior of the same amino acid residues for each of three models were detected and compared. The T-loop locations of the kinase subunit and helix $\alpha 1$ ' of the cyclin domain for three models are presented in Figure 7. The snapshots are displayed for the wild-type Gly16 Arg274 (model I), mutant Ser16 Arg274 (model II), and Gly16 Gln274 (model III) complexes. From the comparison of these snapshots, it is seen that for models
I and III (wild-type Gly16 Arg274 and mutant Gly16 Gln274) the T-loop end is closely positioned to the helix $\alpha 1$ ', while for model II (mutant Ser16 Arg273) formation of a relatively large gap is observed. The gap for the mutant complex Ser16 Arg274 has to remain stable over the long time of dynamical changes. Thus the interaction between the T-loop of the kinase and $\alpha 1$ ' -helix of the cyclin has been observed to be more intense in models I and III than in model II.
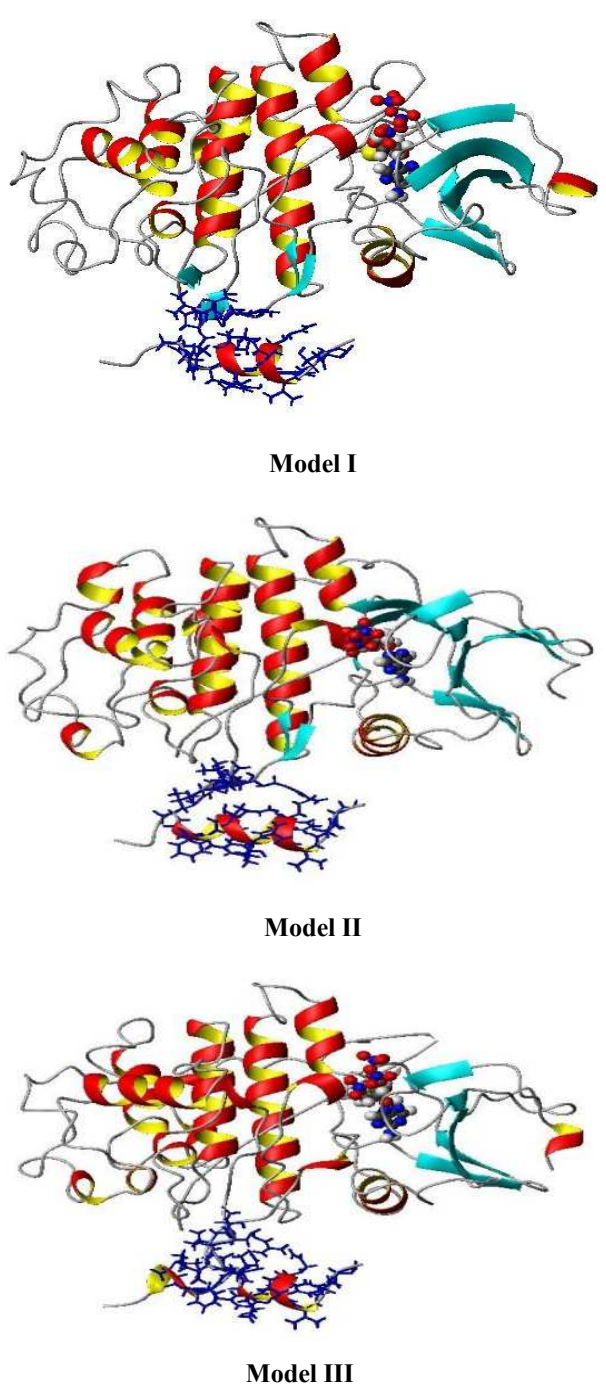

Figure 7. Interaction between the T-loop of the kinase CDK2 and $\alpha 1$ 'helix of cyclin A for mutant Gly16 Arg274, Ser16 Arg274, and Gly16 Gln274 (model I, II, and III, respectively).

To illustrate the interaction between the T-loop and helix $\alpha 1$ ', we calculated the distance between amino acid residue Val155 of the T-loop and residue Thr316 of the helix $\alpha 1$ '. The calculation results are displayed in Figure 8 for three models (blue for model I, red for model II, and dotted lines for model III). The diagrams show the dynamical changes of the distances between the $\mathrm{O}$ atom of the carboxyl group and $\mathrm{N}$ atom of the amino group for residues Val155 and Thr316. The distance between residues Val155 and Thr316 is about 3-4 $\AA$, which makes van der Waals contacts possi- 
ble (Figure 8). It is seen that for models I and III (wild type Gly16 Arg274 and mutant Gly16 Gln274 structures), the distance has to vary greater than for model II (Ser16 Arg274 complex). Thus, for the wild-type Gly16 Arg274 and mutant Gly16 Gln274 proteins, the cyclin helices are more mobile in the T-loop vicinity. It is obvious that for Gly16Ser mutant, such a high mobility of cyclin helices has to disappear. In other words, the Gly16Ser mutation weakens the kinase - cyclin exchange in the vicinity of the activation T-loop region.
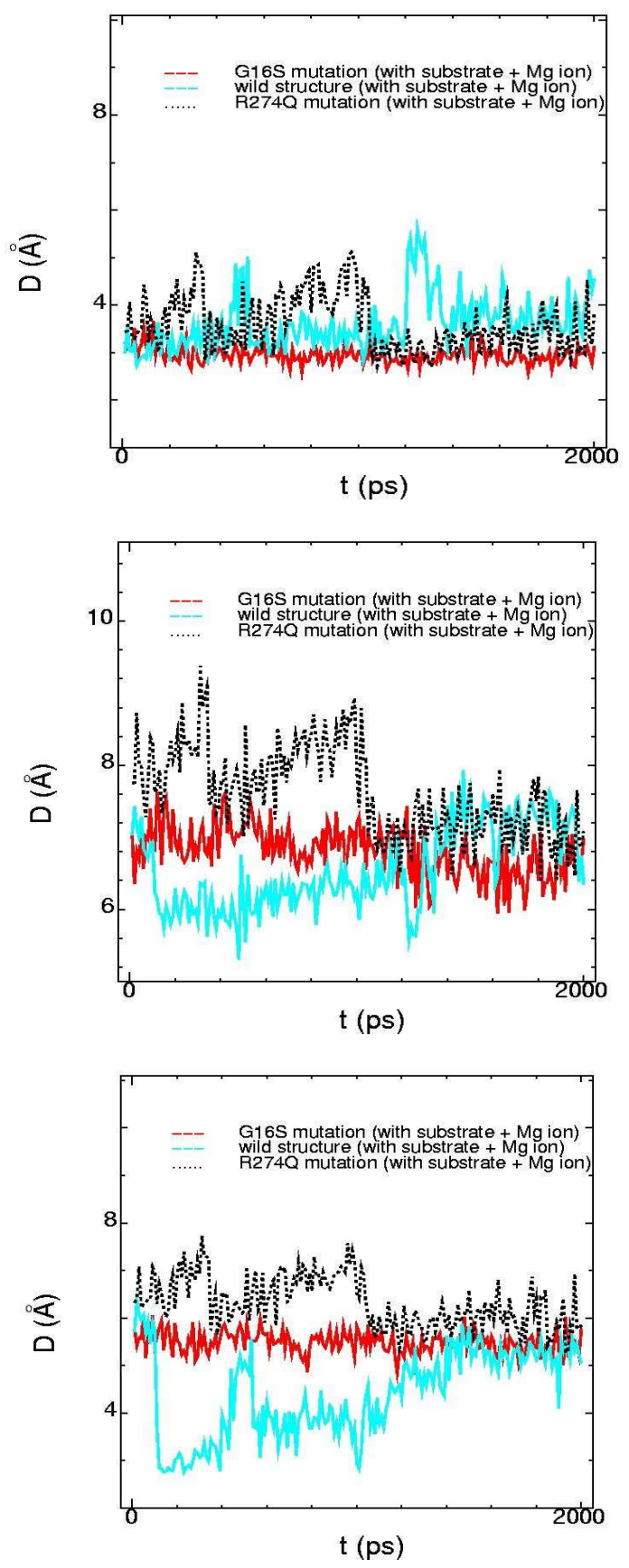

Figure 8. Time dependence of the distances between different atoms of the amino acid residue Val155 of the kinase T-loop and residue Thr316 of the cyclin helix al': Val155 (O) - Thr316 (O) (top); Val155 (N) - Thr316 (N) (middle); Val155 (N) - Thr316 (O) (bottom).
In summary, one of the central problems in understanding the functional and structural properties of a kinase is the interaction of the kinase subunit with a cyclin. CDK2 complexes regulate DNA replication and form complexes with two cyclins: CDK2-cyclin E in G1/S and CDK2-cyclin A in S. Complex CDK2-cyclin A is studied better. At the CDK2-cyclin A interface, the key element is the first repeat of cyclin A, which coincides with the cyclin box [2]. It forms a binding site for the kinase $\alpha 1$ helix, which includes the PSTAIRE sequence (residues 46-56) in the N-terminal $\beta$-sheet, and contacts the T-loop (residues 147-167) of CDK2. To study the details of the CDK2-cyclin A activation mechanism, we have performed a MD simulation of the structure of the $\mathrm{CDK} 2$ - cyclin A interface using the crystal structure of the active kinase complex T160CDK2/cyclin A/ATP- $\mathrm{Mg}^{2+} /$ substrate. Comparisons of CDK2 structural behavior have been made for three kinase complexes (the wild-type, Gly16 $\rightarrow$ Ser, and Arg274 $\rightarrow$ Gln mutant forms). The simulation results show that the above amino acid substitutions can cause considerable conformational changes, especially on the CDK2-cyclin binding sites. We suppose the observed kinase structure peculiarities are responsible for $\mathrm{CDK}$ inactivation and correlate with its degree $[4,11,20-25]$.

\section{The RecA Protein: The Influence of the Gly204 $\rightarrow$ Ser Amino Acid (Mutant) Exchange in Beta-Sheet Loops and DNA Inhibition Sites}

The RecA protein is one of the critical enzymes involved in the SOS mechanism and DNA repair stimulation upon a DNA injury. Genetic recombination includes homologous recombination; affinity to single-strand DNA; induction of the SOS repair system; generation of genetic diversity; preservation of genomic integrity; proper partitioning of chromosomes; and regulation of the cell response to DNA damage. The major central domain of the RecA protein contains the nucleotide binding domain identified by the diffusion of ADP into crystals. Two regions of weak electron density, loop L1 (155-169) and loop L2 (193-207) were supposed to be involved in DNA binding. Based on the homology modeling approach, we have first predicted the conformation of disordered loops L1 and L2. To understand the sensitivity of the artificially generated loops, we have performed MD simulations on the RecA protein to study the influence of amino acid mutations in the beta-sheet and DNA inhibition sites.

During the homology modeling and molecular dynamics (MD) simulations of the RecA protein, we have first created new recA mutants that had mutations in (Gly-204-*Ser) on the secondary structure of the protein. Next, we similarly made a mutation in (Gly-204-*Cys) on the secondary structure of the protein. The Gly204 $\rightarrow$ Ser and Gly204 $\rightarrow$ Cys mutations are known to affect the DNA binding properties. Glycine (Gly) is the smallest amino acid, 
but serine (Ser) is larger. Both amino acids belong to a neutral polar class. They are soluble in water because their polar R-groups may form hydrogen bonds with water (for Gly and Ser polar R-groups, those are $-\mathrm{H}$ and $\mathrm{HO}-\mathrm{CH} 2$, respectively). Cysteine is a polar, neutral, hydrophobic amino acid. Cysteine is one of two sulfur-containing amino acids. Cysteine differs from serine in a single atom: the sulfur of thiol replaces the oxygen of alcohol. Disulfides can form many such protein s and play a key role in their stabilization.

In Figure 9, computer-generated structures of the initial and final configurations (2-ns state) of the recA protein are presented. The drawn bonds in Figure 10 represent the positions of the key amino acid reisdues in the L1 loop: Glu156, Ile157, Gly158, and Gly204 $\rightarrow$ Ser point mutation, respectively. Figure 11 shows the distances between the atomic coordinates for both native and mutant (Gly204 $\rightarrow$ Ser) recA models for the residues around the Gly204 $\rightarrow$ Ser point mutation. The distances clearly differ from each other in their behavior for both the wild and mutant models throughout the whole simulation time from the start to the final (2-ns) state. The Glu156, Ile157, and Gly158 residues lie in the maximal curvature region of the L1 loop. The positional changes are displayed by calculating the distance for both recA proteins - between the wild-type or mutant (Gly204 $\rightarrow$ Ser) and Glu156, Ile157, and Gly158, respectively.
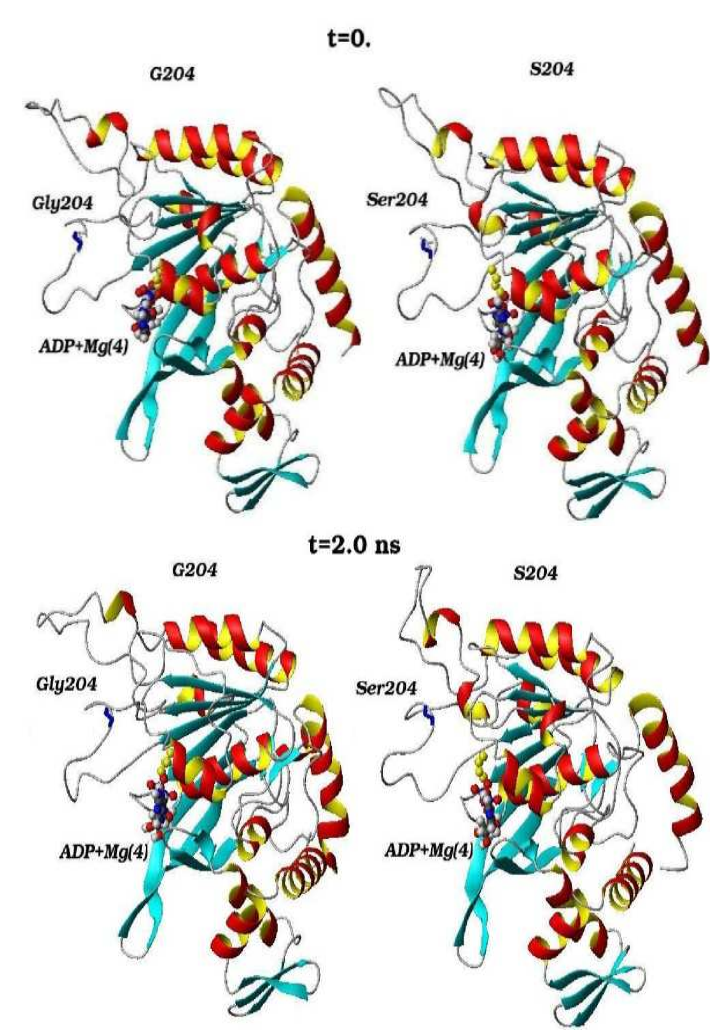

Figure 9. MD-simulated configurations of the wild-type recA (left) and Gly204 $\rightarrow$ Ser mutant (right) proteins. Protein configurations are shown for the initial $(t=0 \mathrm{~ns})($ top) and final $(t=2 \mathrm{~ns})$ (bottom) states.

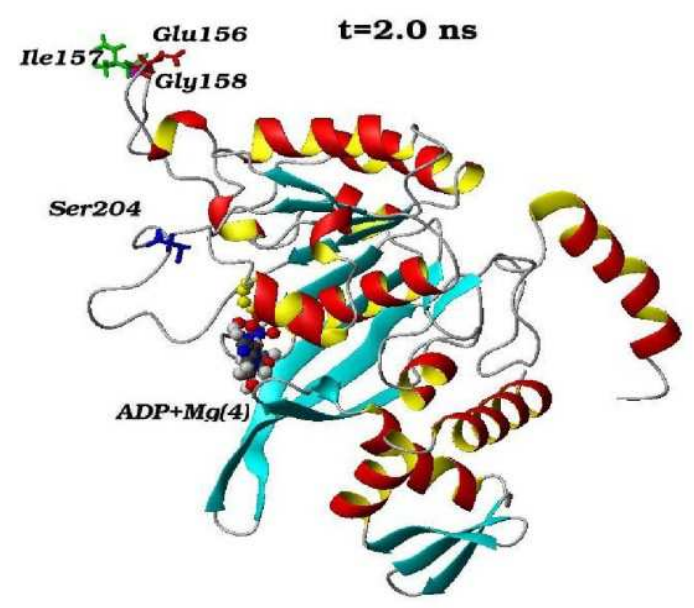

Figure 10. A computer-generated structure of the final configuration ( $t=2$ ns) of the recA protein. The drawn bonds represent the Glu156 (red), Ile157 (green), Gly158 (cyan), and Ser204 point mutation (blue), respectively.

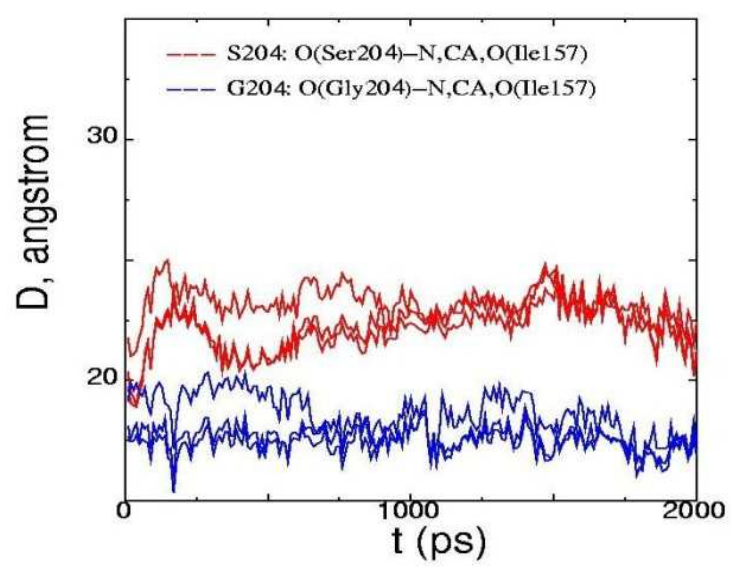

Figure 11. The interatomic distances $(\AA)$ between the atomic coordinates for the residues around the Gly204 $\rightarrow$ Ser point mutation as a function of time (in ps). For the wild-type recA, the distances are shown in blue; for the Gly204 $\rightarrow$ Ser mutant, in red.

In summary, with regard to some aspects of the recA functional properties, the MD simulation results shown in Figures $9-11$ indicate that the chemical change caused by the Gly204 $\rightarrow$ Ser mutation made the disordered L1 loop modify its flexibility. The atomic coordinates were observed to undergo a serious dislocation. In vivo, the rec $\mathrm{A}$ mutation blocks efficient SOS response expression. The region surrounding residue 204 of the RecA protein plays an important role in DNA recognition and binding. The conformational modifications of the protein play a role in direct recombination. Gly204 could equally be involved in a reaction with ATP [26-30].

\section{Appendix. Molecular Dynamics (MD) Simulation Details}

The experimental studies of mutation transitions for protein structures by the traditional X-ray or NMR measurement represent themselves as the extremely difficult, ex- 
pensive and time consuming task. Using the adequate computational methods (molecular dynamics), based on their efficient implementation in the parallel/vector and specialpurpose machines (say, MDGRAPE-2 and 3), would allow ones to simulate the valid behavior of the wild-type and mutated proteins in water or ionic solvents at reliable physiological temperatures and conditions. Thus, the molecular simulation studies between the wild-type and mutant proteins represent themselves as a fundamental problem of great importance.

The principles of force fields (also known as molecular mechanics) are based upon Newtonian mechanics.

Force Field: The basic idea is that bond lengths, valence and torsional angles have "natural" values depending on the involved atoms and that molecules try to adjust their geometries to adopt these values as closely as possible. Additionally, steric and electrostatic interactions, mainly represented by van der Waals and Coulomb forces, are included in the so-called potential. These parameters are optimized to obtain the best of experimental values, as geometries, conformational energies and spectroscopic properties.

Energy calculation includes all contributions:

$$
\mathrm{E}_{\text {total }}=\mathrm{E}_{\text {bond }}+\mathrm{E}_{\text {angle }}+\mathrm{E}_{\text {torsion }}+\mathrm{E}_{\text {non-bonding. }} \text {. }
$$

Many of the molecular modeling force fields in use today can be interpreted in terms of a relatively simple fourcomponent picture of intra- and intermolecular forces within the system.

Bond-Energy: The energy between two bonded atoms increases, when the bond is compressed or stretched. The potential is described by an equation based on Hooke's law for springs.

$$
E_{\text {bond }}=\sum_{\text {bonds }} k_{b}\left(r-r_{0}\right)^{2}
$$

where $\mathrm{kb}$ is the force constant, $\mathrm{r}$ is the actual bond length and $\mathrm{r} 0$ the equilibrium length. This quadratic approximation fails as the bond is stretched towards the point of dissociation.

Angle-Energy: The angular energy increases if the equilibrium bond angles are bent. Again the approximation is harmonic and uses Hooke's law.

$$
E_{\text {angle }}=\sum_{\text {angles }} k_{\theta}\left(\theta-\theta_{0}\right)^{2},
$$

where $\mathrm{k}$ controls the stiffness of the angle; theta is the current bond angle and thetao the equilibrium angle. Both, the force and equilibrium constant have to be estimated for each triple of atoms.

Torsion Energy: Intra-molecular rotations (around torsions or dihedrals) require energy as well.

$$
E_{\text {torsion }}=\sum_{\text {torsions }} \frac{V_{n}}{2}(1+\cos (n \omega-\gamma))
$$

where $\mathrm{V}_{\mathrm{n}}$ controls the amplitude of this periodic function, $\mathrm{n}$ is the multiplicity, and the so-called phase factor, shifts the entire curve along the rotation angle axis z. Again the parameters $\mathrm{V}_{\mathrm{n}} ; \mathrm{n}$ and for all combinations of four atoms have to be determined.

Non-bonding Energy: The simplest potential for nonbonding interactions includes two terms, a Van der Waals and a Coulomb term.

$$
E_{\text {non-bonding }}=\underbrace{\sum_{i} \sum_{j>i}\left(\frac{A_{i j}}{r_{i j}^{6}}-\frac{B_{i j}}{r_{i j}^{12}}\right)}_{\text {Van der Waals }}+\underbrace{\sum_{i} \sum_{j>i} \frac{q_{i} q_{j}}{r_{i j}}}_{\text {Coulomb }},
$$

where the Van der Waals term accounts for the attraction and the Coulomb term for electrostatic interaction. The shown approximation for the van der Waals energy is of the Lennard-Jones 6-12 potential type.

Dynamical equations: Molecular Dynamics employs a technique is so-called united atom method, where atom groups with non-polar hydrogen atoms are treated as an ensemble. The inclusion of the solvent can be done explicitly where the solute is immersed in a cubic box of solvent molecules. The use of non-rectangular periodic boundary conditions, stochastic boundaries and "solvent shell" can help to reduce the number of solvent molecules required and therefore accelerate the MD simulation. When using implicit solvent models in molecular dynamics simulations, there are two additional effects to bear in mind The solvent also influences the dynamical behavior of the solute via (a) random collisions, and by (b) imposing a frictional drag on the motion of the solute through the solvent. While explicit solvent calculations include these effects automatically, it is also possible to incorporate these effects of solvent without requiring any explicit specific solvent molecules to be present. The Langevin equation of motion is the starting point for these stochastic dynamics models:

$$
F_{i}(t)=m_{i} a_{i}(t)=m_{i} \frac{\partial^{2} r_{i}(t)}{\partial t^{2}}
$$

whereas

$$
\begin{gathered}
F_{i}(t)=-\frac{\partial E_{t o t}}{\partial r_{i}} \\
m_{i} \frac{\partial^{2} r_{i}(t)}{\partial t^{2}}=F_{i}\left(r_{i}(t)\right)-\gamma_{i} m_{i} \frac{\partial r_{i}(t)}{\partial t}+R_{i}(t)
\end{gathered}
$$

The first component is due to particle-particle interactions between the. The second force arises from the motion of the particle through the solvent and is equivalent to the frictional drag on the particle due to the solvent. gammai is often referred to as the friction coefficient. The third contribution, the force $\operatorname{Ri}(t)$ is due to random fluctuations caused by interactions with solvent molecules.

\section{Acknowledgement}

This work is a part of our collaboration between JINR 
(Russia), RIKEN (Japan), and Keio University (Japan). The work has been performed within a research collaboration between JINR (Russia) and Keio University (Japan) and was supported by the JSPS (Japan Society for the Promotion of Science)--RFBR (Russian Foundation for Basic Research) (grant No.13-04-92100). The work has been supported in part by the Grant in Aid for the Global Center of Excellence Program to the Center for Education and Research of Symbiotic, Safe and Secure System Design from Japan's Ministry of Education, Culture, Sport, and Technology. The MD simulations have been performed using computer software, hardware facilities, and cluster machines at the CICC (JINR), RICC (RIKEN), and the Yasuoka Laboratory of Keio University (Japan). The authors would like to specially thank Mr. Sergei Negovelov (JINR) for technical assistance and helpful comments.

\section{References}

[1] Vinall R.L, Tepper C.G., Shi X.-B., Xue L.A., Gan-dourEdwards R. and de Vere White R.W., "The R273H p53 mutation can facilitate the androgen-independent growth of LNCaP by a mechanism that involves $\mathrm{H} 2$ re-laxin and its cognate receptor LGR7". Oncogene, 25, 2006, 2082-2093.

[2] Kholmirzo Kholmurodov (Ed.), "Molecular Dynamics of Nanobistructures", Nova Science Publishers Ltd., 2011, 230p., ISBN: 978-1-61324-320-6.

[3] Kholmurodov Kh.T., Fel'dman T.B., and Ostrovskii M.A. "Molecular Dynamics of Rhodopsin and Free Opsin: Computer Simulation". Neuroscience and Behavioral Physiology. 2007. 37(2), 161-174.

[4] Kholmurodov Kh. and Koltovaya N. "Molecular dynamics study of the CDK2-cyclin A interface in the kinase Gly16Ser and Arg274Gln mutants". In: Book of Proceedings of the International Workshop MSSMBS2008 "Molecular Simulation Studies in Material and Biological Sciences, 10-12 September 2008, JINR, Dubna, Russia, by Nova Science Publishers, N.Y.

[5] Pearlman, D.A., Case, D.A., Caldwell, J.W., Ross, W.R., Cheatham, T.E., DeBolt, S., Ferguson, D., Seibel, G., Kollman, P., "AMBER, a computer program for applying molecular mechanics, normal mode analysis, molecular dynamics and free energy calculations to elucidate the structures and energies of molecules". Comp. Phys. Commun., 91, 1995, 141.

[6] Case D.C., Pearlman D.A., Caldwell J.W., Cheatham III T.E., Ross W.S., Simmerling C.L., Darden T.A., Merz K.M., Stanton R.V., Cheng A.L., Vincent J.J., Crowley M., Ferguson D.M., Radmer R.J., Seibel G.L., Singh U.C., Weiner P.K., Kollman P.A., AMBER, 2010.

[7] Cornell, W.D., Cieplak, P., Bayly, C.I., Gould, I.R., Merz, Jr.K.M., Ferguson, D.M., Spellmeyer, D.C., Fox, T., Caldwell, J.W., Kollman, P.A., "A second Generation forth field for the simulation of Proteins and Nucleic Ac-ids". J. Am. Chem. Soc., 117, 1995, 5179-5197.

[8] Humphrey, W., Dalke, A. and Schulten, K., "VMD - Visual Molecular Dynamics". J. Molec. Graphics, 14, 1996, 33-38.
[9] K. Kholmurodov, W. Smith, K. Yasuoka, T. Darden and T. Ebisuzaki, J. Comput. Chem., 21, 2000, 1187, 3.

[10] Kholmurodov Kh.T., Dushanov E.B., Yasuoka K., "MD. Simulations of the P53 oncoprotein structure: the effect of the Arg273 $\rightarrow$ His mutation on the DNA binding domain", Advances in Bioscience and Biotechnology, Volume 2, Number 5, ISSN Print: 2156-8456 ISSN Online: 2156-8502 (2011).

[11] Selwyne R.A., Kholmurodov Kh.T., Koltovaya N.A., "Homology Modelling and Molecular Dynamics of CyclinDependent Protein Kinases", In: IT for Real Rorld Problems, (Universities Press Series in Systems, Models, Informatics and Control), Ed. Sree Hari Rao (Ed.), pp. 1-72.

[12] Kholmurodov Kh.T., Krasavin E.A., Krylov V.A., Dushanov E.B., Korenkov V.V., Yasuoka K., Narumi T., Ohno Y., Taiji M., Ebisuzaki T., "MD Simulations on the Structure of Onco-Proteins P53: Wild-Type and Radioresistant Mutant Systems", In: "MOLECULAR DYNAMICS OF NANOBIOSTRUCTURES", Nova Science Publishers (N.Y.), ISBN: 978-1-61324-320-6, 2011 (in press).

[13] Narumi T., Susukita R., Ebisuzaki T., McNiven G. and Elmergreen B., "Molecular Dynamics Machine: Spe-cialpurpose Computer for Molecular Dynamics Simula-tions". Molecular Simulation, 21, 1999, 401-408.; Narumi T., Susukita R., Furusawa H., Yasuoka K., Kawai A., Koishi T., Ebisuzaki T., MDM version of AMBER, 2000.; Narumi, T., Susukita, R., Furusawa, H., Ebisuzaki, T., "46 Tflops Specialpurpose Computer for Molecular Dynamics Simulations: (WINE-2)". Proc. 5th Int. Conf. on Signal Processing. Beijing., 2000, 575-582; Susukita, R., Ebisuzaki, T., Elmegreen, B.G., Furusawa, H., Kato, K., Kawai, A., Kobayashi, Y., Koishi, T., McNiven, G.D., Narumi, T., Yasuoka, K., "Hardware accelerator for mo-lecular dynamics: MDGRAPE-2", Computer Physics Communications, 155, 115-131(2003).

[14] M. Taiji, T. Narumi, Y. Ohno, N. Futatsugi, A. Suenaga, N. Takada, and A. Konagaya, "Protein explorer: A petaflops spe-cial-purpose computer system for molecular dynamics simulations", in Proceedings of SC2003, Phoenix, AZ 2003; T. Narumi, Y. Ohno, N. Futatsugi, N. Okimoto, A.Suenaga, R.Yanai, and M. Taiji, "A high-speed spe-cial-purpose computer for molecular dynamics simulations: MDGRAPE-3", in Proceedings of NIC Workshop 2006, From Computational Biophysics to Systems Biology, Julich, NIC Series, vol. 34, pp.29- 35, 2006; T. Narumi, Y. Ohno, N. Okimoto, T. Koishi, A. Suenaga, N. Futatsugi, R. Yanai, R. Himeno, S. Fujikawa, M. Ikei, and M. Taiji, "A 185 TFLOPS simulation of amyloid-forming peptides from Yeast prion Sup35 with the special-purpose computer system MDGRAPE-3", in Proceedings of SC2006, Tampa, FL, 2006.

[15] T.P. Sakmar, R.R. Franke, and H.G. Khorana. Glutamic acid-113 serves as the retinylidene Schiff base counterion in bovine rhodopsin, Proc. Natl.Acad. Sci. USA. 1989. 86, 8309-8313.

[16] Hernández-Rodríguez EW, Sánchez-García E, Crespo-Otero R, Montero-Alejo AL, Montero LA, Thiel W. Understanding rhodopsin mutations linked to the retinitis pigmentosa disease: a QM/MM and DFT/MRCI study. //J Phys Chem B. 2012;116(3):1060-76.

[17] Fel'dman T.B., Kholmurodov Kh.T., and Ostrovsky M.A., "Molecular Physiology of Rhodopsin: Computer Simulation". Physics of Particles and Nuclei Letters, 2008, Vol. 5, 
No. 2, pp. 131-144.

[18] Feldman T.B., Kholmurodov Kh.T., Ostrovsky M.A., Khrenova M.G., and Nemukhin A.V., "Studies on the Conformational State of the Chromophore Group (11-cis-Retinal) in Rhodopsin by Computer Molecular Simulation Methods". Biophysics. 2009, Vol. 54, No. 4, pp. 465-470.

[19] Kholmurodov Kh.T., Fel'dman T.B., Ostrovsky M.A., "Interaction of chromophore, $11\ulcorner$ cis $\ulcorner$ retinal, with amino acid residues of the visual pigment rhodopsin in the region of protonated Schiff base: a molecular dynamics study". Russian Chemical Bulletin, International Edition, 2007, Vol. 56, No. 1, pp. 20-27.

[20] De Bondt H.L., Rosenblah J., Jancarik J., Jones H.D., Morgan D.O. and Kim S.-H., "Crystal structure of cyclindependent kinase 2". Nature, 1993, 363: 595-602.

[21] Jeffrey P.D., Russo A.A., Polyak K., Gibbs E., Hurwitz J., Massague J. and Paoletich N.P., "Mechanism of CDK activation revealed by the structure of a cyclin A - CDK2 complex". Nature. 1995, 373: 313-320.

[22] Bártová I., Otyepla M., Křiž Z. and Koča J., "Activation and inhibition of cyclin-dependent kinase- 2 by phosphorylation; a molecular dynamics study reveals the functional importance of the glycine-rich loop". Protein Science, 2006, 13: $1449-1457$.

[23] Kholmurodov Kh.T., Kretov D.A., Gerasimova A.S., and Koltovaya N.A., "Molecular Dynamics Simulation of the Substitution of Serine for the Conserved Glycine in the GLoop in the cdc28-srm Yeast Mutant Using the Crystal Lattice of Human CDK2 Kinase". Biophysics, 2006, Vol. 51, No. 4, pp. 603-614.

[24] Selwyne R.A., Kholmurodov Kh.T., and Koltovaya N.A.,
"Homology Modeling of Yeast Cyclin-Dependent Protein Kinase". Physics of Particles and Nuclei Letters, 2007, Vol. 4, No. 4, pp. 339-342.

[25] Kretov D.A., Kholmurodov Kh.T., and Koltovaya N.A., "An Analysis of ATP Binding with Kinase Catalytic Subunit by Molecular Dynamics Simulation of the CDK2 Active Kinase Crystal Lattice". Russian Journal of Physical Chemistry A, 2007, Vol. 81, No. 10, pp. 1692-1697.

[26] Su Z.Y., Lee W.J., and Wang Y.T., "Target Molecular Simulations of RecA Family Protein Filaments", Int. J. Mol. Sci., 2012, 13, 7138-7148.

[27] Kholmurodov, Kh.T. et.al., "MD Simulations on the Amino Acid (Mutation) Exchanges in RecA Protein Structure", In: "Problems of Biochemistry, Radiation and Space Biology", III International Symposium under the Auspices of UNES$\mathrm{CO}$ dedicated to the centenary of Acad. N.M.Sissakian's birth, 2007, Dubna, 24-28 Jan.

[28] Malkov V.A. and R. D. Camerini-Otero, R.D., "Photocrosslinks between single-stranded DNA and Escherichia coli RecA protein map to loops L1 (amino acid residues 157-164) and L2 (amino acid residues 195-209)", J. Biol. Chem., 1995, 270, 30230 .

[29] Kurumizaka, H., Ikawa S., Sarai A., and Shibata T., "The Mutant RecA Proteins, RecAR243Q and RecAK245N, Exhibit Defective DNA Binding in Homologous Pairing", Arch. Biochem. Biophys., 1999, 365, 83-91.

[30] Kurumizaka, H., Ikawa S., Aihara H., Kashima T., Bazemore L.R., Kawasaki K., Sarai A., Radding C.M. and Shibata T., "Point Mutations in a C-Terminal Domain of RecA Protein That Cause Defective Double-Stranded DNABinding for Homologous Pairing with Single-Stranded DNA" J. Biol. Chem., 1996, 271, 33515-33524. 